\title{
SPECT, CT, and MRI in head injury: acute abnormalities followed up at six months
}

\author{
Anne Mitchener, David J Wyper, James Patterson, Donald M Hadley, J T Lindsay Wilson, \\ Lynette C Scott, Maggie Jones, Graham M Teasdale
}

Institute of

Neurological Sciences,

Southern General

Hospital NHS Trust,

Glasgow

D M Hadley

Department of

Psychology, Stirling

University

J T L Wilson

L C Scott

Correspondence to:

Mrs A Mitchener,

Department of

Neurosurgery, Institute of

Neurological Sciences,

Southern General Hospital

NHS Trust, Glasgow

G51 4TF.

Received 30 June 1994 and in final revised form

12 November 1996

Accepted 12 November 1996

(F Neurol Neurosurg Psychiatry 1997;62:633-636)

Keywords: head injury; SPECT; magnetic resonance imaging; computed tomography; cerebral perfusion

Complex pathophysiological and functional disorders are involved in brain damage after head injury and produce its acute clinical features and the persisting neurological limitations that result in long term disability. Imaging techniques such as CT and MRI can provide knowledge of intracranial anatomical abnormalities, whereas techniques such as single photon emission computed tomography (SPECT) and positron emission tomography

\begin{abstract}
Department of Neurosurgery A Mitchener $M$ Jones

GM Teasdale

Department of Clinical Physics

D J Wyper

J Patterson

Department of

Neuroradiology,

Abstract

Neuroimaging with single photon emission computed tomography (SPECT) using the cerebral blood flow tracer ${ }^{99} \mathrm{Tc}^{\mathrm{m}}$ HMPAO has been used to study acute functional alterations after head injury and residual abnormalities at six month follow up in 32 patients. Comparison has been made with anatomical abnormalities defined acutely with CT and on follow up with MRI. SPECT showed slightly more abnormalities than CT in the acute phase (49 regions of abnormally low tracer uptake on SPECT and 45 lesions on CT). Twenty two of the acute SPECT abnormalities were in normal regions on CT. At follow up MRI showed more abnormalities than SPECT (30 on SPECT and 48 on MRI). Ten of the SPECT deficits were in regions with normal MRI. Comparison of the intensity of late and early SPECT deficits showed that only four early deficits deteriorated whereas 28 improved. Only five of 27 lesions seen on both acute SPECT and CT resolved compared with 16 of 22 lesions seen on SPECT but not on CT. Regions of abnormally high tracer uptake were detected in the acute stage in five of the patients. No high focal uptake was evident on follow up. Ten patients with a residual SPECT abnormality and eight with residual MRI lesions were graded clinically in the upper band of good recovery.
\end{abstract} (PET) can provide insights into the patho- physiological and functional sequelae of injury. Thus SPECT and PET can identify abnormalities of cerebral perfusion which are not disclosed by CT or MRI. ${ }^{1-3}$

Although SPECT with ${ }^{99} \mathrm{Tc}^{\mathrm{m}}-\mathrm{HMPAO}$ does not provide absolute measurements of local cerebral blood flow, the validity of the technique as an indication of relative cerebral perfusion has been established for both normal and abnormal circumstances. Good agreement has been found between the results of autoradiography using ${ }^{99} \mathrm{Tc}^{\mathrm{m}}-\mathrm{HMPAO}$ and iodoantipyrine both in normal ${ }^{4}$ and in ischaemic brain. ${ }^{5}$

Although existing work supports the view that SPECT imaging can provide useful information in acute and late stages of head injury, some questions remain with regard to the natural history of these perfusion deficits and their relation to outcome. Most SPECT studies of patients with head injury have so far not included systematic imaging in both acute and late stages. We report an investigation of patients with head injury using CT (the standard imaging modality in acute head injury) and SPECT in the acute phase. MRI, which is more sensitive than CT to structural alterations, was used along with SPECT at follow up six months after injury.

\section{Patients}

Thirty two patients were investigated (27 men, five women). Each had had a closed head injury and was admitted to the neurosurgical unit within 12 hours of injury. Nine patients were injured in road traffic accidents, 12 in falls, 11 in assaults. Their ages ranged from 18 to 64 with a mean of 31 years. The patients' state when admitted to the neurosurgical unit was assessed with the Glasgow coma scale ${ }^{6}$ and by seeking evidence of focal neurological deficits (for example, hemiparesis, dysphasia). Each subject had lost consciousness at the time of injury. When admitted the conscious level remained impaired in all but two patients, one of whom had a focal neurological deficit, the other a craniofacial fracture. Eight patients had severe injuries, seven moderate, and 17 mild. None of the patients had previously had a head injury or other neurological or psychological disease. 
MRI (left) and SPECT (right) taken four months after injury in a 23 year old man who was the victim of an assault. He had a Glasgow coma scale score of 6 on admission. At the time of scanning his MRI was normal but SPECT showed considerable hypoperfusion bilaterally in the frontal regions. Clinically he was severely disabled at this stage with periods of agitation.

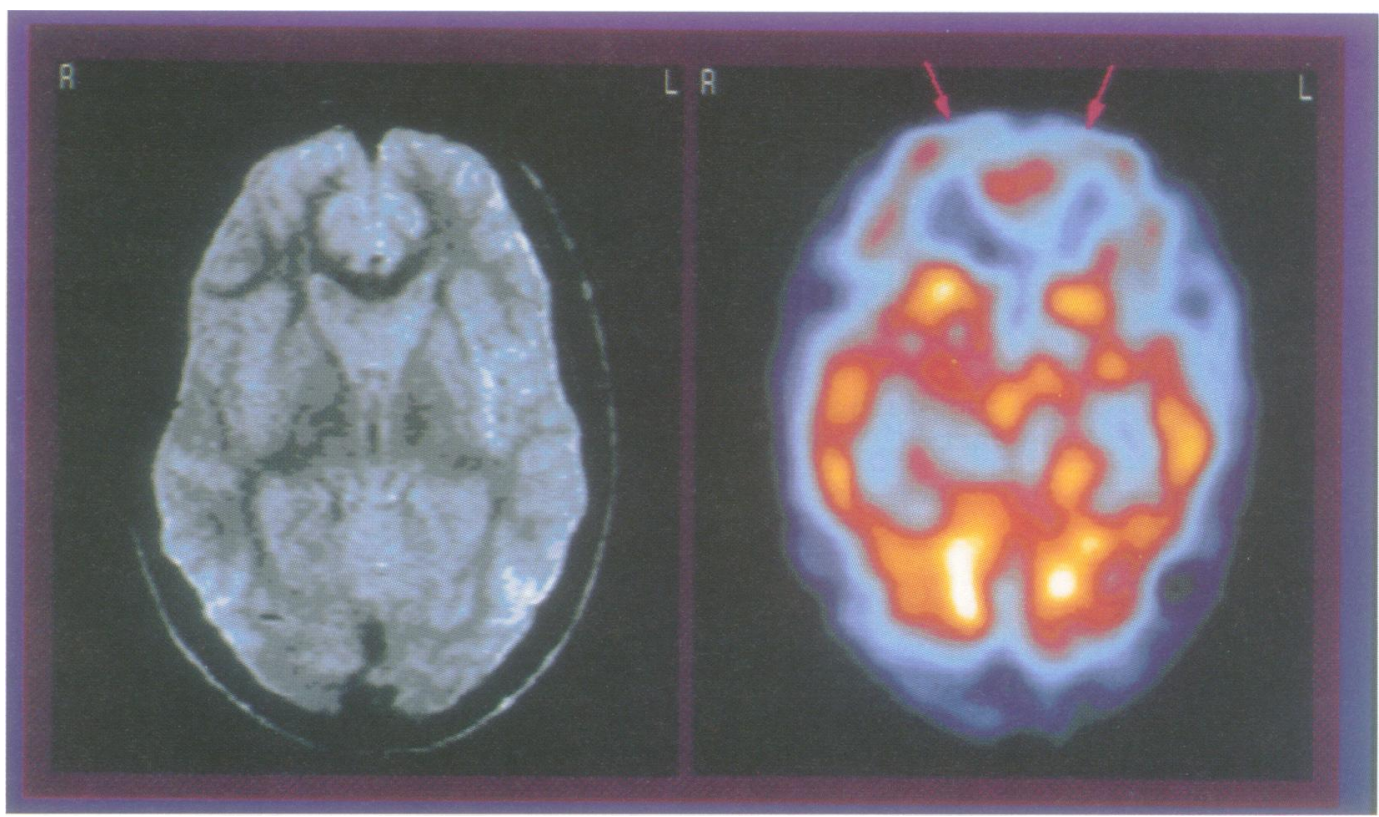

OUTCOME

Patients were reviewed between four and six months after injury. Outcome was assessed according to the Glasgow outcome scale (expanded). ${ }^{7}$ Two patients had severe disability, nine had moderate disability, and 21 made a good recovery.

\section{ACUTE IMAGING}

Each patient had CT performed immediately after arrival at the neurosurgical unit and this was repeated if a patient's clinical condition changed. The images were interpreted by a consultant neuroradiologist who was unaware of the results of SPECT.

Mapping of cerebral blood flow by SPECT was performed using a $500 \mathrm{MBq}$ of ${ }^{99} \mathrm{Tc}^{\mathrm{m}}$ HMPAO (Ceretec) with an SME 810 scanner, between five and nine days after injury. The scanner is a high resolution dedicated head section scanner with transverse resolution of $8 \mathrm{~mm}$ and longitudinal resolution of $12 \mathrm{~mm}$. Typically 16 two minute slices were acquired with a slice separation of $6 \mathrm{~mm}$. On occasions, with more disturbed patients, fewer slices were obtainable. The patients had closed eyes at the time of the injection and there was ambient auditory background. The SPECT scans were read by experienced reporters who were not responsible for patient recruitment and at the time of reporting had no knowledge of the results of other imaging investigations. Reporting was performed at a workstation using SME image display software which enables data to be reoriented in three dimensions and displayed in any plane.

A five point scale was used to classify perfusion as hyperaemic; normal; slightly reduced regional uptake; very reduced regional uptake; or no regional uptake. Originally data were classified into frontal, anterior temporal, posterior temporal, occipital, and parietal regions on the left and right but for this presentation frontal and anterior temporal abnormalities were classed as anterior, and posterior temporal, parietal, and occipital lesions as posterior.
Abnormalities were assessed by comparison with normal scan data and by comparison with the contralateral side. Regions were recorded to be hyperaemic when local uptake of ${ }^{99 \mathrm{~m}} \mathrm{Tc}$ HMPAO was greater than that found in the medial occipital cortex or in a corresponding contralateral region, provided the second was not abnormal.

\section{FOLLOW UP IMAGING}

SPECT was carried out on each patient between four and six months after injury ( 25 at six months). During the same attendance, MRI was performed with a $0 \cdot 15$ Tesla Picker resistive unit using both $\mathrm{T} 1$ and $\mathrm{T} 2$ weighted sequences. The MRI scans were interpreted by a consultant neuroradiologist who was unaware of the results of other studies and clinical findings.

\section{Results}

ACUTE IMAGING

A total of 45 lesions were identified on CT in 27 of the 32 patients; the number of lesions varied from zero to five per patient. The lesions were unilateral in 18 patients and bilateral in nine. Twenty were anterior and 25 were posterior. Two patients had normal SPECT. Their Glasgow coma scale scores on admission were 10 and 11 .

Forty nine areas of perfusion deficit were identified in 30 of the 32 patients. Five patients Thirty of the hypoperfused areas on SPECT were anterior and 19 were posterior. Twenty seven corresponded to focal CT lesions and 22 were in tissue which appeared normal on CT.

\section{FOLLOW UP IMAGING}

A total of 30 SPECT abnormalities were seen; 18 anterior and 12 posterior. Seven patients' scans did not show a localised disturbance of perfusion; these included the two patients whose early scans were normal. No patient was noted to have hyperaemia at follow up. were considered to have areas of hyperaemia. 
Changes in SPECT perfusion deficit between early and late imaging sessions

\begin{tabular}{llll}
\hline $\begin{array}{llll}\text { SPECT perfusion } \\
\text { score }\end{array}$ & 0 & $\begin{array}{l}\text { Considerable } \\
\text { reduction }\end{array}$ & $\begin{array}{l}2 \\
\text { Slight } \\
\text { reduction }\end{array}$ \\
\hline Improved & 5 & 10 & 13 \\
Unchanged & 7 & 7 & 3 \\
Deteriorated & & 2 & 2 \\
\hline
\end{tabular}

The 48 lesions detected by late MRI cannot be directly compared with the results of early $\mathrm{CT}$ in view of the greater sensitivity of the MRI technique. However, 10 of the SPECT abnormalities were in regions that even with MRI appeared structurally normal (figure).

Comparison of the acute and late SPECT imaging showed that of the 49 abnormalities of hypoperfusion seen acutely 28 remained at six months. A further two lesions appeared on late SPECT which were not present on acute scanning. One of these was in a region underlying a subdural haematoma which was hyperperfused in the acute stage. This corresponded with a follow up lesion on MRI. The other was a spreading of frontal hypoperfusion which was normal on follow up MRI. In total seven of the late SPECT abnormalities occurred in areas that were normal on CT in the acute phase and 23 in areas with associated CT lesions.

Lesions that were detected by both CT and SPECT in the early stage were less likely to resolve at follow up (only five of the 27 lesions seen on both acute SPECT and CT resolved compared with 16 of the 22 lesions seen on SPECT only).

The finding of clinical focal hemispheric signs at the time of admission was, in 12 of 14 patients, associated with a perfusion deficit on early scanning, in each case accompanied by a CT abnormality. In 11 of these patients the perfusion abnormality persisted at the late scanning.

Comparison of the intensity of late and early SPECT deficits showed that only four early deficits deteriorated whereas 28 improved by at least one SPECT category (table). Lesions with very low or no initial tracer uptake were least likely to improve.

\section{LATE IMAGING AND OUTCOME}

Six out of seven patients with no late SPECT abnormality had made a good recovery, whereas this occurred in only 15 of 25 patients with one or more lesions. A surprising finding was that eight patients with residual MRI lesions and 10 with late SPECT perfusion deficits (seven corresponding to MRI) had a clinical outcome in the upper band of good recovery.

\section{Discussion}

An individual lesion may be characterised by varying combinations of structural and functional features. Findings on CT are dominated by the high density of haemorrhage with the reduced density of oedema less apparent. Perfusion deficits on SPECT reflect a varying interaction between areas of clot (without perfusion), areas of hypoperfusion as a result of the cerebrovascular effects of injury (compression, spasm etc), and changes in perfusion relating to changes in remote neuronal activity (diaschisis).

The most common pattern of change with time was for improvement, leading to a reduction in the number of lesions demonstrated by SPECT. This was seen most often with lesions that in the acute stage were found only by SPECT and were of mild severity. This raises the speculation that return of perfusion may reflect relief of local vasospasm or reinnervation of functionally deafferented or deactivated areas. The development of new lesions or the worsening of hypoperfusion was generally associated with the demonstration of persisting local structural abnormalities by late MRI. The cause of such deteriorating patterns needs to be investigated.

In this study all scans (SPECT, CT, and MRI) were read by experienced experts-in keeping with conventional reporting practice-rather than analysed by complex computer coregistration software. For this reason the relative extent of lesions on the different modalities has not been considered.

The findings confirm previous reports that SPECT can show hypoperfused tissue which seems normal on structural imaging. ${ }^{1-3}$ SPECT can add to understanding and perhaps to the management of patients with head injury. It adds further information about the pattern of injury in the acute stage and, in particular, provides supportive evidence of diffuse injuries. The different patterns of findings point to lesions in the brain with acute injury being the result of different interactions between structural, vascular, metabolic, and functional processes, perhaps each with different requirements for treatment.

There are reports that disturbances in affect, behaviour, and cognition, which are often present after head injury, are associated with SPECT abnormalities. ${ }^{89}$ Our study has shown that, although on some occasions the presence of lesions on SPECT, MRI, or both can help to explain a poor clinical outcome, it is not necessarily an indication of poor outcome. We have shown that about $30 \%$ of patients were graded in the top band of recovery despite having lesions on SPECT or MRI. This may reflect insensitivity of the clinical outcome scale or indicate that these lesions had minimal functional impact. This is the subject of further investigation.

LCS, and the cost of SPECT and MRI, were supported by the Wellcome Trust.

1 Roper SN, Mena I, King WA, et al. An analysis of cerebral blood flow in acute closed-head injury using technetium $99 \mathrm{~m}$-HMPAO SPECT and computed tomography. $₹ \mathrm{Nucl}$ Med 1991;32:1684-7.

2 Newton MR, Greenwood RJ, Britton KE, et al. A study comparing SPECT with CT and MRI after closed head injury. F Neurol Neurosurg Psychiatry 1992;55:92-4.

3 Gray BG, Ichise M, Chung DG, Kirsh JC, Franks W. Technetium-99m-HMPAO SPECT in the evaluation of patients with a remote history of traumatic brain injury: a comparison with $x$ ray computed tomography. $\mathcal{F}$ Nucl Med 1992;33:52-8.

4 Lear JL. Quantitative local cerebral blood flow measurements with technetium-99m HMPAO: evaluation using multiple radionuclide digital quantitative autoradiogra- 
phy f Nucl Med 1988;29:1387-92.

5 Bullock R, Patterson J, Park C. Evaluation of ${ }^{99 \mathrm{~m} T c-h e x a-}$ methylpropyleneamine oxime cerebral blood flow mapping after acute focal ischemia in rats. Stroke 1991;22 1284-90.

6 Teasdale G, Jennett B. Assessment of coma and impaired consciousness: a practical scale. Lancet 1974;ii:81-4.

7 Jennett B, Snoek J, Bond MR, Brooks N. Disability after severe head injury: observations on the use of the Glasgow outcome scale. ₹ Neurol Neurosurg Psychiatry
$1981 ; 44: 285-93$

8 Oder W, Goldenberg G, Spatt J, Podreka I, Binder H, Deecke $L$. Behavioural and psychosocial sequelae of severe closed head injury and regional cerebral blood flow: a spect study. F Neurol Neurosurg Psychiatry 1992; 55:475-80

9 Goldenberg G, Oder W, Spatt J, Podreka I. Cerebral correlates of disturbed executive function and memory in survivors of severe closed head injury: a spect study. F Neurol Neurosurg Psychiatry 1992;55:362-8.

\section{HISTORICAL NOTES}

\section{Pavlov on neuroimaging}

The work of the Russian physiologist Ivan Petrovich Pavlov (1849-1936) continues to influence modern psychophysiology, neurology, and biological psychiatry. Not generally appreciated, however, is his visionary anticipation of the functional neuroimaging techniques the multicoloured products of which are on the verge of becoming standard decorations for the covers of present day neuroscience journals. In a lecture read before the Society of the Moscow Scientific Institute (March 1913), Pavlov mused about the possibility of localising human brain functions by visualisation of those neuronal structures which are activated during the performance of a particular task. The most relevant passage reads:
"If we could look through the skull into the brain of a consciously thinking person, and if the place of optimal excitability were luminous, then we should see playing over the cerebral surface, a bright spot with fantastic, waving borders constantly fluctuating in size and form, surrounded by a darkness more or less deep, covering the rest of the hemispheres."'

PETER BRUGGER Neurology Clinic, University Hospital Zurich, -8091 Zurich, Switzerland 1 Pavlov IP. Lectures on conditioned reflexes. Twenty-five years of
objective study of the higher nervous activity (behaviour) of animals. London: Martin Lawrence, 1928:222. 